\title{
In situ carcinoma developed over oral lichen planus: a case report with analysis of BUB3, p16, p53, Ki67 and SOX4 expression
}

\author{
Eduardo Augusto ROSA ${ }^{1}$, Erica Negrini LIA², Sergio Bruzadelli MACEDO², Rivadavio Fernandes Batista de AMORIM³
}

\author{
1- Posgraduate Program of Medical Sciences, Faculty of Medicine, University of Brasília, Brasília, DF, Brazil \\ 2- Department of Dentistry, Faculty of Health Sciences, University of Brasilia, Brasília, DF, Brazil \\ 3- Department of Pathology, Faculty of Medicine, University of Brasília, Brasília, DF, Brazil
}

Corresponding address: Erica Negrini Lia - Faculdade de Ciências da Saúde, Departamento de Odontologia - Campus Universitário Darcy Ribeiro Universidade de Brasília - Brasília - DF - Brazil - 70910-900 - Phone (61) 3107-1803 - e-mail: erica.lia@terra.com.br / ericalia@unb.br

Submitted: February 23, 2015 - Modification: July 12, 2015 - Accepted: July 28, 2015

\section{ABSTRACT}

\begin{abstract}
$\mathrm{O}$ ral lichen planus (OLP) represents a common mucocutaneous disease. Various authors have suggested that OLP has malignant potential; however, the mechanisms involved in malignant transformation have not yet been elucidated. A 79-year-old man presented a white lesion for five months in the buccal mucosa diagnosed as OLP. After two months using $0.05 \%$ clobetasol ointment for treatment, the lesion became ulcerated. A new biopsy of the same lesion was performed, and histological analysis showed an in situ oral carcinoma (ISOC). An immunohistochemistry panel was performed, and p16 expression was negative in OLP, however, it showed weak cytoplasmic staining in ISOC. There was strong nuclear BUB3 staining in both OLP and ISOC areas. p53 showed less intense nuclear staining in both regions. Ki67 was negative in OLP area, but showed nuclear staining in the ISOC. SOX4 was negative in both studied areas. BUB3 expression, first reported in this case, and the p16 expression may suggest some influence of these genes on pathogenesis or malignant potential of OLP.
\end{abstract}

Keywords: Carcinoma in situ. Oral lichen planus. Oral pathology. Immunohistochemistry. Neoplastic cell transformation.

\section{INTRODUCTION}

Oral lichen planus (OLP) represents a common mucocutaneous disease that affects 0.5 to $2 \%$ of the population ${ }^{5}$. Malignant transformation of OLP in oral squamous cell carcinoma (OSCC) is possible according to a 10-year retrospective study, besides some cross-sectional and cohort studies ${ }^{4-6,10,29}$. However, the mechanisms involved in malignant transformation have not yet been elucidated; therefore, some authors do not consider that OLP presents this potential ${ }^{3}$.

p53 and Ki67 are current biomarkers used in OSCC and precursor lesions. The establishment of new biomarkers to predict or indicate malignant transformation would be very useful in the management of OLP patients ${ }^{21,22}$. Some genes and antibodies, such as p16, BUB3 and SOX4, are currently studied in different types of cancer in order to establish new possible markers. However, little is known about such antibodies in OSCC precursor lesions or conditions, especially OLP ${ }^{1,8,11,13,27}$.

This paper aims to report a case of an in situ oral carcinoma (ISOC) that developed on a previous OLP lesion in a 79-year-old man. We evaluated the immunohistochemical expression of Ki67, p53, p16, and, for the first time, BUB3 and SOX4.

\section{CASE REPORT}

A 79-year-old white man complained of a white lesion in the buccal mucosa to his dermatologist. He was being monitored in a medical service for older adults for a long time because of multiple basal cell skin carcinomas previously treated. The patient had hypertension treated with captopril and indapamide. He denied smoking and alcoholism. The remaining medical history was not relevant. 


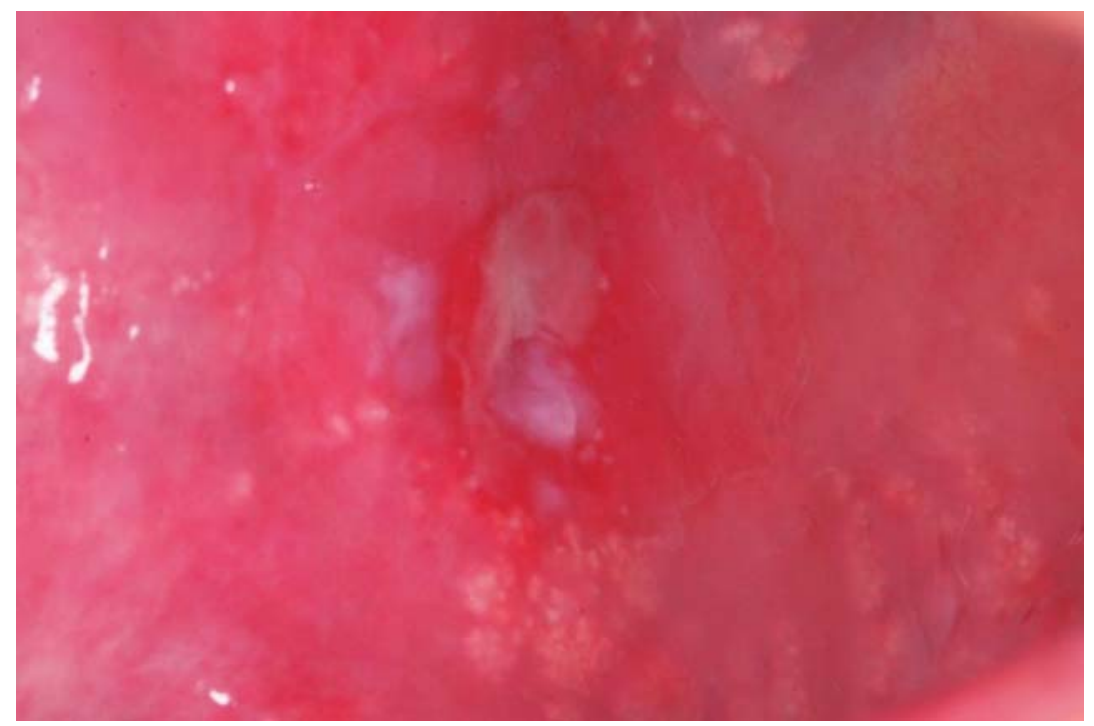

Figure 1- Clinical aspect of the right buccal mucosa: ulcer with an erythematous halo and small white plaques in the center of the picture
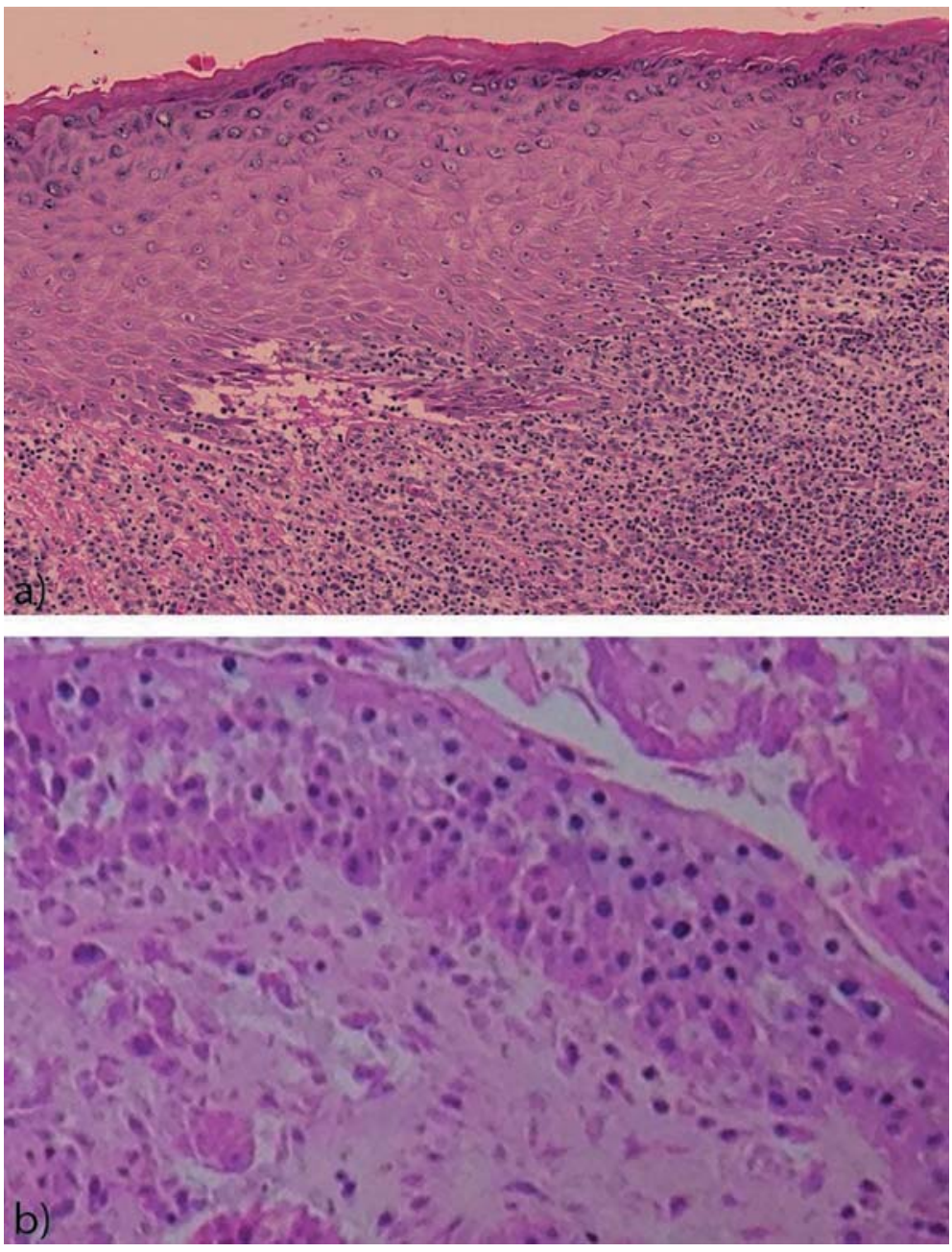

Figure 2- Histological appearance [Hematoxylin \& eosin, 100X (a), 400X (b)]: a) band-like lymphocytic infiltrate in the lichenoid area; b) Carcinoma in situ showing several changes from basal layer to upper layer of epithelium. The following alterations are present: cells with enlarged nuclei, pleomorphic cells, hyperchromatism, increased nuclear-to-cytoplasmic ratio, loss of epithelium stratification and basal cell hyperplasia 

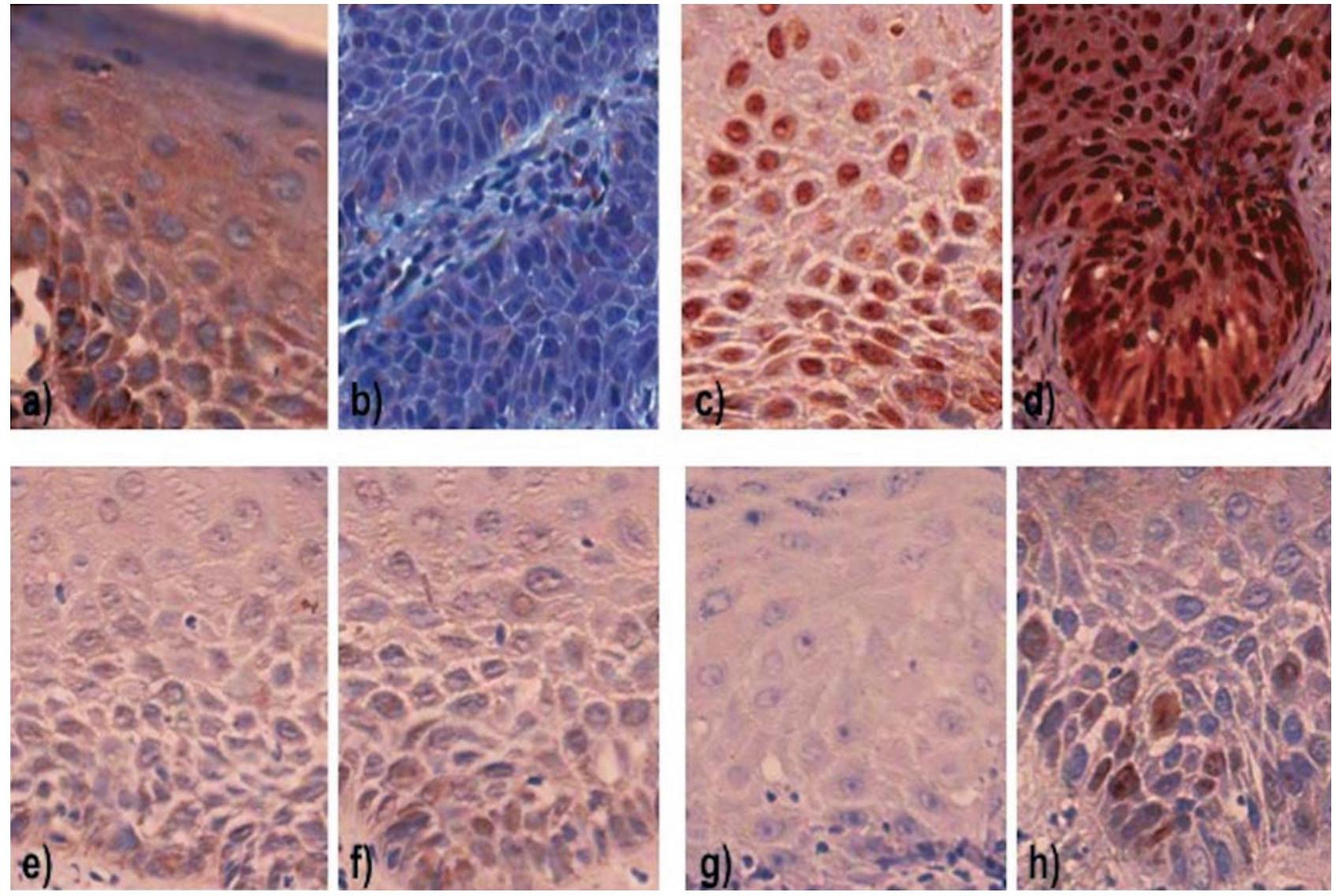

Figure 3- Immunohistochemistry (400X): p16 nuclear and cytoplasmic staining basal and suprabasal layers - oral lichen planus (OLP) area (a); p16 nuclear and cytoplasmic staining basal and suprabasal layers - in situ oral carcinoma (ISOC) area (b); BUB3 nuclear staining - OLP area (c); BUB3 nuclear staining - ISOC area (d); p53 nuclear staining - OLP area (e); p53 nuclear staining - ISOC area (f); Ki67 negative - OLP area (g); Ki67 nuclear staining basal and suprabasal layers - ISOC area (h)

An incisional biopsy of the buccal lesion was conducted by the dermatologist at the right buccal mucosa. Five months after such procedure, the patient became symptomatic, and some erythematous areas appeared mixed with white areas. At that time, the diagnosis of OLP was established by the dermatologist who prescribed $0.05 \%$ clobetasol ointment. After two months without lesion remission, the patient was referred to an oral and maxillofacial surgeon, who found an ulcer with an erythematous halo and some small white plaques around in the right buccal mucosa (Figure 1). Some smaller white plaques were also observed on the left buccal mucosa. A new incisional biopsy was performed by the oral surgeon at the same area of the right-sided lesion, previously diagnosed as OLP.

Histological examination revealed pleomorphic epithelial cells with hyperchromatic nuclei and evident nucleoli. A few mitotic figures and loss of epithelial stratification were also identified. The subjacent connective tissue was not involved. These findings were consistent with in situ oral carcinoma (ISOC). Furthermore, adjacent to the ISOC area, the epithelium showed hyperkeratosis, angulated rete pegs and basal cell liquefaction. Extensive lymphocytic band-like infiltrate was also observed. Altogether, the histological and clinical findings were consistent with the previous diagnosis of OLP. Histological aspects are shown in Figure 2.

An immunohistochemical panel with Ki67 (BIOCARE, SP6, 1:100), p53 (DAKO, Clone DO-7, 1:200), p16 INK4a (Pharmingen, Clone G175405 BD, 1:200), SOX4 and BUB3 (ABCAM, Clone EPR5319[2], 1:500) antibodies was conducted (Figure 3). p16 was positive both in the area of OLP as the ISOC, with cytoplasmic and nuclear staining in the basal and suprabasal layers. BUB3 showed nuclear staining in more than $90 \%$ of cells in both studied areas. p53 showed less than $10 \%$ nuclear staining in the OLP and less than $20 \%$ in the ISOC. SOX 4 was negative in both studied areas. Ki67 showed from 20 to $30 \%$ of positive cells only in the ISOC area. The antibodies data are shown in Table 1.

The lesion was resected with safe surgical margins. Histological examination revealed some areas compatible with ISOC, some compatible with epithelial dysplasia (moderate to severe), and also one area close to the lateral limit of the specimen 
Table 1- Immunohistochemical panel

\begin{tabular}{|c|c|c|c|}
\hline Antibodie & & OLP & ISOC \\
\hline P16 INK4a & $\begin{array}{c}\text { Clone G175-405 BD } \\
\text { Pharmingen }\end{array}$ & $(+)$ & $(+)$ \\
\hline \multirow[t]{3}{*}{$1: 200$} & & Weak and Focal & Weak and Focal \\
\hline & & Cytoplasmatic & Cytoplasmatic \\
\hline & & Basal layer & Basal layer \\
\hline BUB 3 & Clone EPR5319(2) & $(+)$ & $(+)$ \\
\hline \multirow[t]{3}{*}{$1: 500$} & ABCAM & Strong $(>90 \%)$ & Strong (> 90\%) \\
\hline & & Nuclear & Nuclear \\
\hline & & $\begin{array}{l}\text { Basal, espinous and } \\
\text { granular layers. }\end{array}$ & $\begin{array}{c}\text { Basal, espinous and } \\
\text { granular layers. }\end{array}$ \\
\hline P 53 & Clone DO-7 & $(+)$ & $(+)$ \\
\hline \multirow[t]{3}{*}{$1: 200$} & DAKO & Weak <10\% & Weak $<20 \%$ \\
\hline & & Nuclear & Nuclear \\
\hline & & Basal layer & Basal and spinous layers \\
\hline sox 4 & Policlonal & $(-)$ & $(-)$ \\
\hline $1: 800$ & ABCAM & & \\
\hline Ki67 & Clone SP6 & $(-)$ & $(+)$ \\
\hline \multirow[t]{3}{*}{$1: 100$} & BIOCARE & & Strong 20 a $30 \%$ \\
\hline & & & Nuclear \\
\hline & & & Basal and spinous layers \\
\hline
\end{tabular}

$\mathrm{OLP}=$ oral lichen planus; ISOC $=$ in situ oral carcinoma

compatible with OLP. No signs of microinvasion were identified. Four months after surgery, the patient had scarring in the buccal mucosa, but no signs of recurrence were observed. No syndrome associated with basal cell carcinoma was detected.

\section{DISCUSSION}

OLP can manifest as whitish striations or plaques, atrophic erythematous areas, ulcers, papules and vesicles. Three morphological types are usually described: the reticular form - asymptomatic whitish striations; the erythematous or atrophic form - reddish areas alone or between whitish striations, and the erosive form - painful ulcerations added to the other features. The predominant morphology can change over time. The present case is compatible with the erosive form that has the highest probability of malignant transformation ${ }^{4,8}$. However, lichenoid reaction to captopril could also be considered, which represents a limitation in the present case.

In a prospective study conducted with 327 OLP patients, eight of them (2.4\%) developed OSCC ${ }^{4}$. Although the sample had 229 women and only 98 men, the rate of transformation of OLP to OSCC was higher in men $(3 \%)$ than in women $(2.1 \%)$. The mean age in patients who developed OSCC was 68 years in women and 62 years in men, both higher than the overall group ${ }^{4}$. Our patient was even older than the female median in comparison with this group. Moreover, some authors indicate that that OLP can evolve both in squamous cell carcinoma and, possibly, oral melanoma.

Timing for carcinoma appearance in preexisting OLP in the same study varied from 2.68 to 3.26 years ${ }^{4}$. However, time between OLP diagnosis and development of malignancy in the current case was only seven months. It is possible that OLP was already present for much longer as a reticular form without symptoms.

The patient was being monitored in a medical service for older adults for a long time before complaining of mouth problems. Nevertheless, he was only referred to the dentist after an attempt to treat the oral problems. We consider that the mouth exam should be performed preemptively by a dentist, aiming to achieve early diagnosis and avoid complications. A large study conducted with 3142 patients found that $1 \%$ had oral potentially malignant disorders, among them 9 cases of OLP $^{26}$. The importance of oral analysis and tactile examination in earlier diagnosis of oral mucosal diseases was emphasized. 
Multiple basal cell carcinomas may be a component of several syndromes such as GorlinGoltz $^{20}$. No keratocysts or other phenotypes of this syndrome were detected by the dental team. In the same way, no other syndromes were detected by the dermatologists in the previous exam.

Higher expression of Ki67 and p53 in OLP compared with normal oral mucosa was previously observed, which may favor the malignant potential of OLP2. p53 is an important tumor suppressor gene related to oral carcinogenesis. Its mutations have been detected in OLP and OSCC among other lesions, and may be related to malignancy development ${ }^{18,19,25}$. In the present case, although weak, there was p53 marking in both ISOC and OLP areas, confirming the potential of malignancy associated with the OLP. Ki67 antibody is widely used to assess cellular proliferation in cancer and cancer precursor lesions including oral lesions ${ }^{21,22}$. There was no considerable expression of $\mathrm{Ki} 67$ in the OLP area, different from what was observed in some previous studies, in which the expression ranged from 13 to $20 \%{ }^{2,15,30}$. This suggests that some cases of OLP may present a potential for malignant transformation independent of Ki67 expression.

p16 is an INK4a family member with a role in cell cycle regulation. Its function leads to inhibition of $\mathrm{Rb}$ phosphorylation, arresting cell cycle ${ }^{17}$. Suppression of p16 expression appears to represent an initial event in oral carcinomas development ${ }^{9}$. However, similar to cervical intraepithelial neoplasia and cervical cancer, p16 overexpression was also found in OSCC ${ }^{1,9}$. The HPV inhibition of Rb protein leads to high p16 levels, due to negative feedback regulation in cervical lesions. Thus, p16 is considered a useful biomarker for cervical cancer?

p16 expression in OLP was evaluated in three previous studies, with positivity index ranging from 26.7 to $65.2 \% \%^{23,24}$. However, the criteria used in each study was very different ( $\mathrm{p} 16$ expression $>1 \%,>5 \%$ and $>70 \%$ to be a positive case). Normal mucosa and OSCC were 16 negative ${ }^{16}$. In the present case, the p16 expression was found in focal areas, both in OLP and ISOC. This expression, similarly to cervical carcinoma, may reflect the malignant potential of OLP or simply suggest the presence of HPV in OLP. More studies are needed to elucidate the relationship between OLP and $\mathrm{p} 16$.

Numerous cell cycle regulatory molecules have been studied recently. BUB3 has an important role in stabilizing the spindle assembly checkpoint. Some studies using different molecular biology techniques have correlated deregulation of BUB3 expression in different types of cancer $^{8}$. The low expression of BUB3 can lead to abnormal chromosome segregation and allow specific oncogenic mutations to appear ${ }^{12}$. However, overexpression of BUB3 gene was also correlated with high expression of $\mathrm{Ki} 67$ in gastric cancer ${ }^{11}$

In a similar pattern, an intense epithelial expression of BUB3 was detected in the present case, indicating that BUB3 may behave in the same way in oral cancer and precursor lesions as OLP. Since this is a pioneer report of BUB3 expression in an oral lesion, scientific studies with appropriate design should be conducted to confirm that hypothesis.

SOX4 is a molecule involved in DNA transcription and regulation of apoptosis. Increased expression of SOX4 has been detected in malignant tumors of breast, lung, colon and salivary glands, among others ${ }^{14}$. A study with 50 cases of OSCC showed a significant correlation between the expression of SOX4 and tumor staging ${ }^{28}$. The expression of SOX4 in poorly differentiated OSCC was higher than that observed in well-differentiated tumors. Furthermore, a high expression of SOX4 in lymph node metastases was also observed. The authors suggested that SOX4 may have a role in the differentiation of oral cancer. However, SOX4 was negative in the present case. There are no studies evaluating the immunohistochemical expression of SOX4 in OLP lesions so far in scientific literature to compare the present finding.

\section{CONCLUSION}

The present case supports the malignant potential of OLP through the clinical course. In addition, the pattern of Ki67 (absent) and p53 (weak) expression in OLP areas suggest that even in presence of typical histological appearance, OLP can undergo malignant transformation. These findings emphasize the utmost importance of early diagnosis and continuous monitoring of OLP lesions, especially in older patients. BUB3 expression, first reported in the present case, and p16 may suggest some influence of these genes on the pathogenesis or the malignant potential of OLP. More detailed studies should be performed to investigate such hypotheses.

\section{REFERENCES}

1- Abrahao AC, Bonelli BV, Nunes FD, Dias EP, Cabral MG. Immunohistochemical expression of p53, p16 and hTERT in oral squamous cell carcinoma and potentially malignant disorders. Braz Oral Res. 2011;25(1):34-41.

2- Acay RR, Felizzola CF, Araújo NS, Sousa SO. Evaluation of proliferative potential in oral lichen planus and oral lichenoid lesions using immunohistochemical expression of p53 and Ki67. Oral Oncol. 2006;42:475-80.

3- Accurso BT, Warner BM, Knobloch TJ, Weghorst CM, Shumway BS, Allen CM, et al. Allelic imbalance in oral lichen planus and assessment of its classification as a premalignant condition. Oral Surg Oral Med Oral Pathol Oral Radiol Endod. 2011;112:359-66. 
4- Bombeccari GP, Guzzi G, Tettamanti M, Giannì AB, Baj A, Pallotti $F$, et al. Oral lichen planus and malignant transformation: a longitudinal cohort study. Oral Surg Oral Med Oral Pathol Oral Radiol Endod. 2011;112:328-34.

5- Carbone M, Arduino PG, Carrozzo M, Gandolfo S, Argiolas MR, Bertolusso $G$, et al. Course of oral lichen planus: a retrospective study of 808 northern Italian patients. Oral Dis. 2009;15:235-43. 6- Casparis S, Borm JM, Tektas S, Kamarachev J, Locher MC, Damerau G, et al. Oral lichen planus (OLP), oral lichenoid lesions (OLL), oral dysplasia, and oral cancer: retrospective analysis of clinicopathological data from 2002-2011. Oral Maxillofac Surg. 2015;19:149-56.

7- Cheah PL, Looi LM, Teoh KH, Mun KS, Nazarina AR. p16(INK4a) is a useful marker of human papillomavirus integration allowing risk stratification for cervical malignancies. Asian Pac J Cancer Prev. 2012;13(2):469-72.

8- De Voer RM, Geurts van Kessel A, Weren RD, Ligtenberg MJ, Smeets D, Fu L, et al. Germline mutations in the spindle assembly checkpoint genes BUB1 and BUB3 are risk factors for colorectal cancer. Gastroenterology. 2013;145(3):544-7.

9- Dragomir LP, Simionescu C, Mărgăritescu A, Stepan A, Dragomir IM, Popescu MR. P53, p16 and Ki67 immunoexpression in oral squamous carcinomas. Rom J Morphol Embryol. 2012;53:89-93. 10 - Eisen D. The clinical features, malignant potential, and systemic associations of oral lichen planus: a study of 723 patients. J Am Acad Dermatol. 2002;46:207-14.

11- Grabsch H, Takeno S, Parsons WJ, Pomjanski N, Boecking A, Gabbert HE, et al. Overexpression of the mitotic checkpoint genes BUB1, BUBR1, and BUB3 in gastric cancer-association with tumour cell proliferation. J Pathol. 2003;200(1):16-22.

12- Hernando E, Orlow I, Liberal V, Nohales G, Benezra R, Cordon-Cardo $\mathrm{C}$. Molecular analyses of the mitotic checkpoint components hsMAD2, hBUB1 and hBUB3 in human cancer. Int J Cancer. 2001;95(4):223-7.

13- Jafarnejad SM, Wani AA, Martinka M, Li G. Prognostic significance of Sox4 expression in human cutaneous melanoma and its role in cell migration and invasion. Am J Pathol. 2010;177(6):2741-52.

14- Lin CM, Fang CL, Hseu YC, Chen CL, Wang JW, Hsu CL, et al. Clinical and prognostic implications of transcription factor SOX4 in patients with colon cancer. PLoS One. 2013;8(6):e67128.

15- Montebugnoli L, Farnedi A, Marchetti C, Magrini E, Pession A, Foschini MP. High proliferative activity and chromosomal instability in oral lichen planus. Int J Oral Maxillofac Surg. 2006;35(12):1140-4.

16- Montebugnoli L, Venturi M, Gissi DB Leonardi E, Farnedi A, Foschini MP. Immunohistochemical expression of p16INK4A protein in oral lichen planus. Oral Surg Oral Med Oral Pathol Oral Radiol Endod. 2011;112:222-7.
17- Namazie A, Alavi S, Olopade OI, Pauletti G, Aghamohammadi N, Ahamohammadi M. Cyclin D1 amplification and p16(MTS1/ CDK4I) deletion correlate with poor prognosis in head and neck tumors. Laryngoscope. 2002;112(3):472-81.

18- Ogmundsdóttir HM, Björnsson J, Holbrook WP. Role of TP53 in the progression of pre-malignant and malignant oral mucosal lesions. A follow-up study of 144 patients. J Oral Pathol Med. 2009;38(7):565-71.

19- Ogmundsdóttir HM, Hilmarsdóttir $H$, Astvaldsdóttir A, Jóhannsson $\mathrm{JH}$, Holbrook WP. Oral lichen planus has a high rate of TP53 mutations. A study of oral mucosa in Iceland. Eur J Oral Sci. 2002;110(3):192-8.

20- Pastorino L, Pollio A, Pellacani G, Guarneri C, Ghiorzo P, Longo $\mathrm{C}$, et al. Novel PTCH1 mutations in patients with keratocystic odontogenic tumors screened for nevoid basal cell carcinoma (NBCC) syndrome. PLoS ONE. 2012;7(8):e43827.

21- Perisanidis C, Perisanidis B, Wrba F, Brandstetter A, El Gazzar $\mathrm{S}$, Papadogeorgakis $\mathrm{N}$, et al. Evaluation of immunohistochemical expression of p53, p21, p27, cyclin D1, and Ki67 in oral and oropharyngeal squamous cell carcinoma. J Oral Pathol Med. 2012;41(1):40-6.

22- Pitiyage G, Tilakaratne WM, Tavassoli M, Warnakulasuriya S. Molecular markers in oral epithelial dysplasia: review. J Oral Pathol Med. 2009;38(10):737-52.

23- Poomsawat S, Buajeeb W, Khovidhunkit SO, Punyasingh J. Overexpression of cdk4 and $\mathrm{p} 16$ in oral lichen planus supports the concept of premalignancy. J Oral Pathol Med. 2011;40(4):294-9. 24- Salehinejad J, Sharifi N, Amirchaghmaghi M, Ghazi N, Shakeri MT, Ghazi A. Immunohistochemical expression of $\mathrm{p} 16$ protein in oral squamous cell carcinoma and lichen planus. Ann Diagn Pathol. 2014;18(4):210-3.

25- Venza M, Catalano T, Visalli M, Venza I, Lentini M, Curia MC, et al. Association of the DSS1 c.143G >A polymorphism with skin squamous cell carcinoma. J Invest Dermatol. 2010;130(6):171925.

26- Villa A, Gohel A. Oral potentially malignant disorders in a large dental population. J Appl Oral Sci. 2014;22(6):473-6.

27- Wang C, Zhao H, Lu J, Yin J, Zang L, Song N, et al. Clinicopathological significance of SOX4 expression in primary gallbladder carcinoma. Diagn Pathol. 2012;7:41.

28- Watanabe M, Ohnishi, Y, Wato M, Tanaka A, Kakudo K. SOX4 expression is closely associated with differentiation and lymph node metastasis in oral squamous cell carcinoma. Med Mol Morphol. 2014;47(3):150-5.

29- Xue JL, Fan MW, Wang SZ, Chen XM, Li Y, Wang L. A clinical study of 674 patients with oral lichen planus in China. J Oral Pathol Med. 2005;34:467-72.

30- Zargaran M, Jamshidi S, Eshghyar N, Moghimbeigi A. Suitability/unsuitability of cell proliferation as an indicator of malignant potential in oral lichen planus: an immunohistochemical study. Asian Pac J Cancer Prev. 2013;14(11):6979-83. 